\section{JTI}

JOURNAL OF

TRAUMA AND INJURY

\title{
Immediate Post-laparotomy Hypoten- sion in Patients with Severe Traumatic Hemoperitoneum
}

Gil Jae Lee, M.D., Min A Lee, M.D., Byungchul Yoo, M.D., Youngeun Park, M.D., Myung Jin Jang, M.S., Kang Kook Choi, M.D.

Department of Traumatology, Gachon University College of Medicine, Gachon University Gil Medical Center, Incheon, Korea

Received: March 20, 2020

Revised: March 23, 2020

Accepted: March 24, 2020

\section{Correspondence to}

Kang Kook Choi, M.D.

Department of Traumatology, Gachon University College of Medicine, Gachon University Gil Medical Center, 783 Namdong-daero, Namdong-gu, Incheon 21556, Korea

Tel: $+82-32-460-3010$

Fax: +82-32-460-2372

E-mail:choikangkook@gilhospital.com

This paper was presented at 2017 5th Pan Pacific Trauma Congress.

Purpose: Immediate post-laparotomy hypotension (PLH) is a precipitous drop in blood pressure caused by a sudden release of abdominal tamponade after laparotomy in cases of severe hemoperitoneum. The effect of laparotomy on blood pressure in patients with significant hemoperitoneum is unknown.

Methods: In total, 163 patients underwent laparotomy for trauma from January 1, 2013 to December 31, 2015. Exclusion criteria included the following: negative laparotomy, only a hollow viscous injury, and hemoperitoneum $<1,000 \mathrm{~mL}$. After applying those criteria, 62 patients were enrolled in this retrospective review. PLH was defined as a decrease in the mean arterial pressure (MAP) $\geq 10 \mathrm{mmHg}$ within 10 minutes after laparotomy.

Results: The mean estimated hemoperitoneum was 3,516 mL. The incidence of PLH was $23 \%$ (14 of 62 patients). The MAP did not show significant differences before and after laparotomy ( 5 minutes post-laparotomy, $67.5 \pm 16.5$ vs. $68.3 \pm 18.8 \mathrm{mmHg} ; p=0.7$; 10 minutes post-laparotomy, $67.5 \pm 16.5$ vs. $70.4 \pm 18.8 \mathrm{mmHg} ; p=0.193)$. The overall in-hospital mortality was $24 \%$ ( 15 of 62 patients). Mortality was not significantly higher in the PLH group (two of 14 [14.3\%] vs. 13 of 48 [27.1\%]; $p=0.33$ ). No statistically significant between-group differences were observed in the intensive care unit and hospital stay.

Conclusions: PLH may be less frequent and less devastating than it is often considered. Surgical hemostasis during laparotomy is important. Laparotomy with adequate resuscitation may explain the equivalent outcomes in the two groups.

Keywords: Hypotension; Wounds and injuries; Shock; Intra-abdominal hypertension; Hemorrhage 


\section{INTRODUCTION}

Blood pressure management is crucial during surgery, as intraoperative hypotension can cause severe complications, such as myocardial infarction and stroke [1-4]. Immediate post-laparotomy hypotension (PLH) is a precipitous drop in blood pressure caused by the sudden release of abdominal tamponade after laparotomy in cases of severe hemoperitoneum. It is a feared phenomenon by anesthesiologists, and the concerns of some anesthesiologists regarding PLH may delay emergency laparotomy until they are able to prepare more blood for transfusion and other equipment. Adequate preoperative preparation and resuscitation require time and effort. In these cases, we must decide whether to open the abdomen immediately to stop the bleeding or to wait for the blood to be transfused. The most common cause of shock in trauma is hemorrhage [5-11]. Therefore, controlling the source of bleeding is an essential and urgent component of trauma care. However, the effect of laparotomy on blood pressure in patients with significant hemoperitoneum is unknown. This study aimed to elucidate the frequency of PLH and to determine the effect of laparotomy to blood pressure in cases of significant hemoperitoneum.

\section{METHODS}

A retrospective cohort study was performed, involving a total of 163 patients who underwent laparotomy for trauma at the Gil Medical Center which is the regional trauma center of Incheon, Korea from January 1, 2013 to December 31,2015. Patients with a negative laparotomy, hollow viscous injury, and hemoperitoneum $<1,000 \mathrm{~mL}$ were excluded from this study because these conditions do not lead to any significant increase in intra-abdominal pressure. After applying the above criteria, 62 patients were ultimately enrolled in the current study. PLH was defined as a decrease in the mean arterial pressure (MAP) $\geq 10$ $\mathrm{mmHg}$ within 10 minutes after laparotomy. The MAP at 5 minutes before laparotomy was compared to the MAP at 5 and 10 minutes after laparotomy. We divided the enrolled patients into two groups (PLH and non-PLH) and compared variables between the groups. The primary outcome of the study was in-hospital mortality, and the secondary outcome was intensive care unit (ICU) and hospital stay.

Continuous data were presented as means \pm standard deviation. Differences in nominal and categorical variables were compared using the Fisher's exact test, while the Mann-Whitney $U$ test was utilized to compare differences between continuous variables. The MAP readings before and after laparotomy were compared using the paired $t$-test. Binary logistic regression was used in the multivariate analysis. A two-tailed $p$-value $<0.05$ was considered to indicate statistical significance. All statistical analyses were performed with SPSS version 19.0 for Windows (IBM Corp., Armonk, NY, USA).

\section{RESULTS}

The mean estimated hemoperitoneum was $3,516 \mathrm{~mL}$. The incidence of PLH was $23 \%$ ( 14 of 62 patients) when the preoperative MAP was compared with the MAP at 5 minutes after laparotomy. When we compared the preoperative MAP with the MAP at 10 minutes after laparotomy, the incidence was $26 \%$ (16 of 62 patients). The incidence of MAP elevation was $60 \%$ (37 of 62 patients) at 5 minutes and 63\% (39 of 62 patients) at 10 minutes after laparotomy.

When we defined PLH as a decrease in MAP at 5 minutes after laparotomy, among the variables that we investigated, the initial MAP of the PLH group was significantly higher than that of the non-PLH group (82.0 \pm 18.4 vs. $64.6 \pm 17.6 \mathrm{mmHg} ; p=0.006)$, as illustrated in Table 1 . The initial lactate level of the PLH group was also higher than that of the non-PLH group ( $7.8 \pm 5.0$ vs. $4.9 \pm 3.5 \mathrm{mmol} / \mathrm{L}$; $p=0.046)$, as presented in Table 1 .

The MAP before laparotomy was not significantly different from the MAP after laparotomy at either time point ( 5 minutes after laparotomy, $67.5 \pm 16.5$ vs. $68.3 \pm 18.8 \mathrm{mmHg} ; p=0.7 ; 10$ minutes after laparotomy, $67.5 \pm 16.5$ vs. $70.4 \pm 18.8 \mathrm{mmHg} ; p=0.193)$.

The overall in-hospital mortality rate was $24 \%$ (15 of 62 patients). PLH did not significantly increase mortality (two of 14 [14.3\%] with PLH vs. 13 of 48 [27.1\%] without PLH; $p=0.33$ ) (Table 2). No statistically significant be- 
tween-group differences were observed in ICU and hospital stay (Table 2). The multivariate analysis showed that PLH was not an adverse factor associated with in-hospital mortality (Table 3).

\section{DISCUSSION}

The current study was designed to examine the incidence of PLH and the effect of laparotomy to patients with significant intra-abdominal bleeding. Depending on one's point of view on PLH, there may be a conflict of opinion

Table 1. Comparison of demographic and physiologic variables between the PLH and non-PLH groups

\begin{tabular}{|lccl|}
\hline & PLH (n=14) & $\begin{array}{c}\text { Non-PLH } \\
(\mathbf{n}=\mathbf{4 8})\end{array}$ & $\boldsymbol{p}$-value \\
\hline Age (years) & $50.7 \pm 20.3$ & $51.6 \pm 17.2$ & 0.86 \\
Male/female ratio & $0.64(9 / 14)$ & $0.89(41 / 48)$ & 0.008 \\
Blunt mechanism & 81.2 & 76.1 & 0.745 \\
Inotropic agent & $100(14 / 14)$ & $82.6(38 / 48)$ & 0.08 \\
Injury severity score & $23.3 \pm 10.8$ & $22.1 \pm 11.1$ & 0.73 \\
RTS & $7.1 \pm 1.2$ & $6.3 \pm 1.3$ & 0.12 \\
RBC transfusion (pack) & $3.9 \pm 2.1$ & $4.3 \pm 3.5$ & 0.61 \\
Initial MAP (mmHg) & $82.0 \pm 18.4$ & $64.6 \pm 17.6$ & 0.006 \\
Initial Hb (g/dL) & $9.7 \pm 3.4$ & $11.2 \pm 3.0$ & 0.1 \\
pH & 7.0 & $7.0 \pm 0.1$ & 0.55 \\
Lactate (mmol/L) & $7.8 \pm 5.0$ & $4.9 \pm 3.5$ & 0.046 \\
\hline
\end{tabular}

Values are presented as mean \pm standard deviation or number (\%). PLH: post-laparotomy hypotension, RTS: Revised Trauma Score, RBC: red blood cell, MAP: mean arterial pressure, Hb: hemoglobin.

Table 2. Comparison of outcomes between the PLH group (at 5 minutes after the laparotomy) and the non-PLH group

\begin{tabular}{|lccc|}
\hline & PLH $(\mathbf{n}=\mathbf{1 4})$ & $\begin{array}{c}\text { Non-PLH } \\
(\mathbf{n = 4 8})\end{array}$ & $\boldsymbol{p}$-value \\
\hline Mortality rate & $14.3(2 / 14)$ & $27.1(13 / 48)$ & 0.33 \\
ICU stay (day) & $9.0 \pm 11.0$ & $10.9 \pm 19.7$ & 0.725 \\
Hospital stay (day) & $18.0 \pm 13.3$ & $30.4 \pm 36.2$ & 0.053 \\
\hline
\end{tabular}

Values are presented as mean \pm standard deviation unless otherwise indicated.

PLH: post-laparotomy hypotension, ICU: intensive care unit. between the anesthesiologist and the surgeon, but little evidence exists regarding this issue. To the best of our knowledge, this is first study to explore the incidence of PLH.

We had to define PLH, due to the lack of consensus in the literature. We chose a definition of PLH in this study as a decrease in the MAP of $\geq 10 \mathrm{mmHg}$ within 10 minutes after laparotomy, because the MAP within 10 minutes after laparotomy would reflect its effect better than other possible definitions.

Although no statistical significance was observed, in the present study, it was found that the MAP did not decrease but rather increased at both 5 minutes and 10 minutes after laparotomy. This observation is counterintuitive, but a plausible explanation draws upon the pathophysiology and management of abdominal compartment syndrome (ACS) [12,13]. A variety of factors, ranging from medical to surgical, can cause intra-abdominal hypertension (IAH), but one of the most important causes observed in trauma patients is intra-abdominal hemorrhage [12]. In particular, in cases of hemoperitoneum caused by arterial bleeding, IAH can soon become aggravated, progressing to ACS. Once a patient develops ACS, more complex events occur, including a sudden collapse of venous return that aggravates hypotension, ultimately resulting in a vicious cycle [12]. It is likely that a patient whose abdomen is distended due to hemoperitoneum has already fallen into ACS, in which case the

Table 3. Multivariate analysis of in-hospital mortality after laparotomy

\begin{tabular}{|lccc|}
\hline & OR & 95\% Cl & $p$-value \\
\hline PLH & 2.4 & $0.2-36.2$ & 0.51 \\
EBL & 1.0 & $0.9-1.0$ & 0.17 \\
Mechanism & 0.6 & $0.04-10.6$ & 0.78 \\
Initial MAP & 1.0 & $0.97-1.08$ & 0.34 \\
Hb & 1.2 & $0.8-1.7$ & 0.24 \\
Lactate & 1.0 & $0.6-1.6$ & 0.88 \\
ISS & 0.9 & $0.8-1.0$ & 0.18 \\
Transfusion & 1.0 & $0.6-1.6$ & 0.78 \\
\hline
\end{tabular}

OR: odds ratio, Cl: confidence interval, PLH: post-laparotomy hypotension, EBL: estimated blood loss, MAP: mean arterial pressure, Hb: hemoglobin, ISS: Injury Severity Score. 
appropriate management strategy is laparotomy $[12,13]$. Once laparotomy is performed, the restored venous return compensates for the sudden release of tamponade $[14,15]$.

The serum lactate level of the PLH group was significantly higher than that of non-PLH group. Furthermore, although the difference was not statistically significant, the PLH group had lower initial hemoglobin levels than the non-PLH group. This might suggest that the physiological condition of the PLH group was poorer than that of the non-PLH group. The initial MAP of the PLH group was higher than that of the non-PLH group, which might have been caused by more the frequent use of inotropic agents in the PLH group than in the non-PLH group. Interestingly, even though there were no significant differences, the outcomes analyzed in this study-including mortality rate, ICU stay, and hospital stay-were better in the PLH group than in the non-PLH group. We could not determine whether this result was caused by coincidence, so a follow-up study should be conducted with a larger number of patients.

There are limitations of this study. First, this study was retrospective in nature. There could not be a thorough evaluation of changes in the amount of inotrophic agent use and fluid/blood infusion during the peri-laparotomy period in the current study. Second, the intra-abdominal pressure could not be estimated. Routinely checking the intra-abdominal pressure in patients with hemoperitoneum would be very helpful for evaluating our hypothesis.

\section{CONCLUSION}

In conclusion, PLH may be less frequent and less devastating than it is often considered. Surgical hemostasis duri ng laparotomy is important, as is preparation to manage PLH in patients with significant hemoperitoneum. The effect of this preparation on outcomes requires further study. Laparotomy with adequate resuscitation may explain the equivalent outcomes in the two groups.

\section{REFERENCES}

1. van Waes JA, van Klei WA, Wijeysundera DN, van Wolfswinkel
L, Lindsay TF, Beattie WS. Association between intraoperative hypotension and myocardial injury after vascular surgery. Anesthesiology 2016;124:35-44.

2. Limburg M, Wijdicks EF, Li H. Ischemic stroke after surgical procedures: clinical features, neuroimaging, and risk factors. Neurology 1998;50:895-901.

3. Poulton T, Murray D; National Emergency Laparotomy Audit (NELA) project team. Pre-optimisation of patients undergoing emergency laparotomy: a review of best practice. Anaesthesia 2019;74 Suppl 1:100-7.

4. Marsden M, Carden R, Navaratne L, Smith IM, Penn-Barwell JG, Kraven LM, et al. Outcomes following trauma laparotomy for hypotensive trauma patients: a UK military and civilian perspective. J Trauma Acute Care Surg 2018;85:620-5.

5. Cottingham CA. Resuscitation of traumatic shock: a hemodynamic review. AACN Adv Crit Care 2006;17:317-26.

6. Kauvar DS, Wade CE. The epidemiology and modern management of traumatic hemorrhage: US and international perspectives. Crit Care 2005;9(Suppl 5):S1-9.

7. Baker CC, Oppenheimer L, Stephens B, Lewis FR, Trunkey DD. Epidemiology of trauma deaths. Am J Surg 1980;140:144-50.

8. Acosta JA, Yang JC, Winchell RJ, Simons RK, Fortlage DA, Hollingsworth-Fridlund $\mathrm{P}$, et al. Lethal injuries and time to death in a level I trauma center. J Am Coll Surg 1998;186:52833.

9. Champion HR, Copes WS, Sacco WJ, Lawnick MM, Keast SL, Bain LW Jr, et al. The major trauma outcome study: establishing national norms for trauma care. J Trauma 1990;30:135665.

10. Sauaia A, Moore FA, Moore EE, Moser KS, Brennan R, Read RA, et al. Epidemiology of trauma deaths: a reassessment. J Trauma 1995;38:185-93.

11. Potenza BM, Hoyt DB, Coimbra R, Fortlage D, Holbrook T, Hollingsworth-Fridlund P; Trauma Research and Education Foundation. The epidemiology of serious and fatal injury in San Diego County over an 11-year period. J Trauma 2004;56:6875 .

12. Hunt L, Frost SA, Hillman K, Newton PJ, Davidson PM. Management of intra-abdominal hypertension and abdominal compartment syndrome: a review. J Trauma Manag Outcomes 2014;8:2.

13. Lee RK. Intra-abdominal hypertension and abdominal compartment syndrome: a comprehensive overview. Crit Care Nurse 2012;32:19-31. 
14. Wauters J, Claus P, Brosens N, McLaughlin M, Hermans G, Malbrain M, et al. Relationship between abdominal pressure, pulmonary compliance, and cardiac preload in a porcine model. Crit Care Res Pract 2012;2012:763181.
15. Maffongelli A, Fazzotta S, Palumbo VD, Damiano G, Buscemi S, Maione $\mathrm{C}$, et al. Abdominal compartment syndrome: diagnostic evaluation and possible treatment. Clin Ter 2020;171:e156-60. 